\title{
Dimensional reduction of quantum fields on a brane
}

\author{
Z. Haba \\ Institute of Theoretical Physics, University of Wroclaw, \\ 50-204 Wroclaw, Plac Maxa Borna 9, Poland \\ e-mail:zhab@ift.uni.wroc.pl
}

\begin{abstract}
If we restrict a quantum field defined on a regular $D$ dimensional curved manifold to a $d$ dimensional submanifold then the resulting field will still have the singularity of the original $D$ dimensional model. We show that a singular background metric can force the restricted field to behave as a $d$ dimensional quantum field.
\end{abstract}

\section{$1 \quad$ Introduction}

Quantum fields are defined by their correlation functions. The Lagrangian serves as a heuristic tool for a construction of quantum fields. A reduction of the number of coordinates in the $D$ dimensional Lagrangian does not mean that if we had a complete $D$-dimensional quantum field theory then we could reduce it in any way to a model resembling a quantum field theory in $d<D$ dimensions. We can see this problem already at the level of a massless free field $\phi$. The vacuum correlation function of $\phi(\mathbf{x}(1))$ and $\phi(\mathbf{x}(2))$ is $|\mathbf{x}(1)-\mathbf{x}(2)|^{-D+2}$. If we restrict the field to the hypersurface $x_{D}=0$ setting in all correlation functions $x_{D}(j)=0$ then we obtain a quantum field with a continuous mass spectrum in $d=D-1$ dimensions but this will not be the canonical free field in $d$ dimensions whose two-point function behaves as $|\mathbf{x}(1)-\mathbf{x}(2)|^{-D+3}$ at short distances. Nevertheless, it is an attractive idea that the Universe once had more dimensions and subsequently through a dynamical process shrank to a lower dimensional hypersurface. The dynamics could have the form of a gravitational collapse ( say a ball collapsing to a disk). At the level of field correlation functions this would mean that we have initially scalar, electromagnetic and gravitational fields in $D$-dimensions with their standard canonical singularities which subsequently evolve into fields with $d<D$ dimensional singularity. We show that such a reduction of dimensions is possible when the metric becomes singular. A similar mechanism is suggested in the brane scheme of refs.[1][2]. In ref.[1] 
the authors derive the Green's function in $D=5$ dimensional space-time which on the $d=D-1=4$ submanifold has the singularity of the fourdimensional Green's function. Their model encounters some difficulties when generalized to arbitrary $D$ and $d[3]$. Some other brane-type models of quantum fields are discussed in refs.[4] [5][6][7]. In this letter we discuss a general metric which has power-law singularity. In general relativity such metrics could describe collapse phenomena [8]. We can obtain metrics with power-law singularities as solutions to higher dimensional supergravity theories [9]. These solutions describe $m$ branes or intersecting m-branes in an $m+n$ dimensional space-time [10]).

\section{A quantum field on a D-1 dimensional hyper- surface}

We consider a submanifold $\mathcal{M}_{D-1}$ of a Riemannian manifold $\mathcal{M}_{D}$ whose metric becomes singular near $\mathcal{M}_{D-1}$. The metric on $\mathcal{M}_{D}$ close to $\mathcal{M}_{D-1}$ (in local coordinates) is described by a "warp factor" $a\left(x_{D}\right)$ which becomes singular either when $x_{D} \rightarrow 0$ or $\left|x_{D}\right| \rightarrow \infty$

$$
d s^{2} \equiv g_{\mu \nu} d x^{\mu} d x^{\nu}=d x_{D}^{2}+a\left(x_{D}\right)^{2}\left(d x_{1}^{2}+\ldots .+d x_{D-1}^{2}\right)
$$

The Green's function of the minimally coupled scalar field is a solution of the equation

$$
\mathcal{A} G=g^{-\frac{1}{2}} \delta
$$

where $\mathcal{A}$ is the Laplace-Beltrami operator

$$
\mathcal{A}=g^{-\frac{1}{2}} \partial_{\mu}\left(g^{\mu \nu} g^{\frac{1}{2}} \partial_{\nu}\right)
$$

In the metric (1) eq.(2) reads

$$
\left(\partial_{D} a^{D-1} \partial_{D}+a^{D-3} \triangle\right) G=\delta\left(x_{D}-x_{D}^{\prime}\right) \delta\left(\mathbf{x}-\mathbf{x}^{\prime}\right)
$$

where $d=D-1, \mathbf{x}=\left(x_{1}, \ldots, x_{d}\right)$ and $\triangle$ is the $d$-dimensional Laplacian. This equation is simplified if we introduce the coordinate

$$
\eta=\int a^{-d} d x_{D}
$$

Then

$$
\left(\partial_{\eta}^{2}+a^{2 d-2} \triangle\right) G=\delta\left(\eta-\eta^{\prime}\right) \delta\left(\mathbf{x}-\mathbf{x}^{\prime}\right)
$$

In the paper of Dvali et al $[1] D=4$ and $a^{4}\left(x_{D}(\eta)\right) \rightarrow \delta(\eta)$.

We discuss in detail the case

$$
a\left(x_{D}\right)=\left|x_{D}\right|^{\alpha}
$$


Then

$$
\left(\partial_{D}\left|x_{D}\right|^{\alpha d} \partial_{D}+\left|x_{D}\right|^{\alpha(d-2)} \triangle\right) G=\delta\left(x_{D}-x_{D}^{\prime}\right) \delta\left(\mathbf{x}-\mathbf{x}^{\prime}\right)
$$

We define

$$
\eta=|1-\alpha d|^{-1} x_{D}\left|x_{D}\right|^{-\alpha d}
$$

then eq.(8) takes the form

$$
\left(\partial_{\eta}^{2}+\kappa|\eta|^{2 \nu} \triangle\right) G=\delta\left(\eta-\eta^{\prime}\right) \delta\left(\mathbf{x}-\mathbf{x}^{\prime}\right)
$$

or in terms of the Fourier transform $\tilde{G}$ in $\mathbf{x}$

$$
\left(\partial_{\eta}^{2}-\mathbf{p}^{2} V(\eta)\right) \tilde{G}=\delta\left(\eta-\eta^{\prime}\right)
$$

where

$$
V(\eta)=\kappa|\eta|^{2 \nu}
$$

with

$$
\nu=\alpha(d-1)(1-\alpha d)^{-1}
$$

and

$$
\kappa=|1-\alpha d|^{-2 \nu}
$$

Eq.(11) can be solved by means of the Feynman-Kac integral applying the proper time method

$$
\begin{aligned}
& G\left(\eta, \mathbf{x} ; \eta^{\prime}, \mathbf{x}^{\prime}\right)=\frac{1}{2}(2 \pi)^{-d} \int_{0}^{\infty} d \tau \int d \mathbf{p} \exp \left(i \mathbf{p}\left(\mathbf{x}^{\prime}-\mathbf{x}\right)\right) \\
& E\left[\delta\left(\eta^{\prime}-\eta-b(\tau)\right) \exp \left(-\frac{1}{2} \mathbf{p}^{2} \int_{0}^{\tau} V(\eta+b(s)) d s\right)\right]
\end{aligned}
$$

Here, $b(s)$ is the Brownian motion [11] defined as the Gaussian process with the covariance

$$
E[b(s) b(t)]=\min (s, t)
$$

$E[.$.$] denotes an average over the paths of the Brownian motion.$

The dimensional reduction is imposed by setting $\eta=\eta^{\prime}=0$. Next, we use the equivalence $b(s)=\sqrt{\tau} b\left(\frac{s}{\tau}\right)$ which follows from eq.(15). Then, using the scaling invariance of the potential $\mathrm{V}$ (i.e., $V(\lambda \eta)=\lambda^{2 \nu} V(\eta)$ ) we have

$$
\begin{aligned}
& G\left(0, \mathbf{x} ; 0, \mathbf{x}^{\prime}\right)=\frac{1}{2}(2 \pi)^{-d} \int_{0}^{\infty} d \tau \int d \mathbf{p} \exp \left(i \mathbf{p}\left(\mathbf{x}^{\prime}-\mathbf{x}\right)\right) \\
& E\left[\delta(\sqrt{\tau} b(1)) \exp \left(-\frac{1}{2} \tau^{1+\nu} \mathbf{p}^{2} \int_{0}^{1} V(b(s)) d s\right)\right]
\end{aligned}
$$

Changing the variables

$$
\mathbf{p}=\tau^{-\frac{1}{2}-\frac{\nu}{2}} \mathbf{k}
$$

and

$$
\tau=r\left|\mathbf{x}-\mathbf{x}^{\prime}\right|^{\frac{2}{1+\nu}}
$$

we obtain

$$
G\left(0, \mathbf{x} ; 0, \mathbf{x}^{\prime}\right)=C\left|\mathbf{x}^{\prime}-\mathbf{x}\right|^{-d+\frac{1}{1+\nu}}
$$


with a certain constant $C$. If $0>\nu>-1$ then the singularity of the Green's function is weaker than the one for the $D$-dimensional free field. The Green's function is equal to the Green's function of the $d=D-1$ dimensional free field if $\nu=-\frac{1}{2}$ what corresponds to $\alpha=\frac{1}{2-d}$. The potential with $2 \nu=-1$ has the same scaling dimension as $V=\delta(\eta)$ applied by Dvali et al [1]. The Hamiltonian with the potential $V(\eta)=|\eta|^{-1}$ and the path integral (16) require a careful definition if $2 \nu \leq-1$ but at least till $2 \nu \geq-2$ such a definition (through a regularization and a subsequent limiting procedure) is possible [12]. Eq.(11) with the $\delta$-potential (" $\delta$-brane") also involves a particular regularization and its subsequent removal [13]. Let us consider a solution of this problem by means of the proper time method. The heat kernel $K^{\delta}$ is known exactly for the $\delta$-potential [14]. Hence,

$$
\begin{aligned}
& G\left(\eta, \mathbf{x} ; \eta^{\prime}, \mathbf{x}^{\prime}\right)=\frac{1}{2}(2 \pi)^{-4} \int_{0}^{\infty} d \tau \int d \mathbf{p} \exp \left(i \mathbf{p}\left(\mathbf{x}^{\prime}-\mathbf{x}\right)\right) K^{\delta}\left(\eta, \eta^{\prime}, \tau\right) \\
& =\frac{1}{2}(2 \pi)^{-4} \int_{0}^{\infty} d \tau \int d \mathbf{p} \exp \left(i \mathbf{p}\left(\mathbf{x}^{\prime}-\mathbf{x}\right)\right) \\
& \left(K_{0}\left(\eta-\eta^{\prime}, \tau\right)-2 \mathbf{p}^{2} \int_{0}^{\infty} d u \exp \left(-2 \mathbf{p}^{2} u\right) K_{0}\left(|\eta|+\left|\eta^{\prime}\right|+u, \tau\right)\right)
\end{aligned}
$$

where

$$
K_{0}(\eta, \tau)=(2 \pi \tau)^{-\frac{1}{2}} \exp \left(-\frac{1}{2 \tau} \eta^{2}\right)
$$

is the heat kernel for the Brownian motion.

When $\eta=\eta^{\prime}=0$ the $\tau$-integral of the first term on the r.h.s. of eq.(18) (the one independent of $\mathbf{p}$ ) is infinite (and proportional to $\delta\left(\mathbf{x}-\mathbf{x}^{\prime}\right)$ ) whereas the second integral gives the formula(17) with $\nu=-\frac{1}{2}$.

Eq.(18) could have been derived as a limiting case of eqs. (6) and(16) when $a\left(x_{D}\right)^{2 d-2} \rightarrow \delta(\eta)$. On the Lagrangian level we have

$$
\int d x_{D} d \mathbf{x} \sqrt{g} g^{D D} \partial_{D} \phi \partial_{D} \phi=\int d \eta d \mathbf{x} \partial_{\eta} \phi \partial_{\eta} \phi
$$

and

$$
\int d x_{D} d \mathbf{x} \sqrt{g} g^{j k} \partial_{j} \phi \partial_{k} \phi=\int d \eta d \mathbf{x} a^{2 d-2} \partial_{j} \phi \partial_{j} \phi \rightarrow \int d \eta d \mathbf{x} \delta(\eta) \partial_{j} \phi \partial_{j} \phi
$$

Hence, we recover the Lagrangian of Dvali et al [1].

\section{A generalization to surfaces of arbitrary di- mensions}

Let us consider on a $D=m+n$ dimensional manifold a metric (in local coordinates) which close to the $n$-dimensional surface takes the form

$$
d s^{2}=|\mathbf{y}|^{2 \beta} d \mathbf{y}^{2}+|\mathbf{y}|^{2 \alpha} d \mathbf{x}^{2}
$$


where $\mathbf{y} \in R^{m}$ and $\mathbf{x} \in R^{n}$. Eq.(2) for the Green's function of the LaplaceBeltrami operator reads

$$
\left(\frac{\partial}{\partial y^{i}}|\mathbf{y}|^{\beta(m-2)+\alpha n} \frac{\partial}{\partial y^{i}}+|\mathbf{y}|^{\beta m+\alpha(n-2)} \frac{\partial}{\partial x^{j}} \frac{\partial}{\partial x^{j}}\right) G_{E}=\delta
$$

We discuss here only a simplified form of eq.(23) which appears when

$$
\beta(m-2)+\alpha n=0
$$

In such a case eq.(23) reads

$$
\left(\frac{\partial}{\partial y^{i}} \frac{\partial}{\partial y^{i}}+|\mathbf{y}|^{2 \beta-2 \alpha} \frac{\partial}{\partial x^{j}} \frac{\partial}{\partial x^{j}}\right) G_{E}=\delta
$$

or taking the Fourier transform in $\mathbf{x}$

$$
\left(\frac{\partial}{\partial y_{i}} \frac{\partial}{\partial y_{i}}-\mathbf{p}^{2} V(\mathbf{y})\right) \tilde{G}_{E}=\delta(\mathbf{y})
$$

We obtain again an equation for the Green's function of the Schrödinger operator with the potential

$$
V(\mathbf{y})=|\mathbf{y}|^{2 \beta-2 \alpha}
$$

and the coupling constant $\mathbf{p}^{2}$. We solve eq.(26) by means of the proper time method

$$
\begin{aligned}
& G\left(\mathbf{y}, \mathbf{x} ; \mathbf{y}^{\prime}, \mathbf{x}^{\prime}\right)=\frac{1}{2}(2 \pi)^{-n} \int_{0}^{\infty} d \tau \int^{\tau} d \mathbf{p} \exp \left(i \mathbf{p}\left(\mathbf{x}^{\prime}-\mathbf{x}\right)\right) \\
& E\left[\delta\left(\mathbf{y}^{\prime}-\mathbf{y}-\mathbf{b}(\tau)\right) \exp \left(-\frac{1}{2} \mathbf{p}^{2} \int_{0}^{\tau} V(\mathbf{y}+\mathbf{b}(s)) d s\right)\right]
\end{aligned}
$$

where $\mathbf{b}$ is the $m$-dimensional Brownian motion.

On the brane $\mathbf{y}=\mathbf{y}^{\prime}=\mathbf{0}$. In such a case using $\mathbf{b}(s)=\sqrt{\tau} \mathbf{b}\left(\frac{s}{\tau}\right)$ we have

$$
\int_{0}^{\tau} d s V(\mathbf{b}(s))=\tau^{1+\beta-\alpha} \int_{0}^{1} V(\mathbf{b}(s)) d s
$$

Hence, if we change variables

$$
\mathbf{p}=\mathbf{k} \tau^{-\frac{1}{2}(1+\beta-\alpha)}
$$

then

$$
\begin{aligned}
& G\left(\mathbf{0}, \mathbf{x} ; \mathbf{0}, \mathbf{x}^{\prime}\right) G\left(\mathbf{0}, \mathbf{x} ; \mathbf{0}, \mathbf{x}^{\prime}\right) \\
& =\frac{1}{2}(2 \pi)^{-n} \int_{0}^{\infty} d \tau \bar{\tau}^{n(\alpha-1-\beta)-m} \int d \mathbf{k} \exp \left(i \mathbf{k} \sqrt{\tau}^{\alpha-1-\beta}\left(\mathbf{x}^{\prime}-\mathbf{x}\right)\right) \\
& E\left[\delta(\mathbf{b}(1)) \exp \left(-\frac{1}{2} \mathbf{k}^{2} \int_{0}^{1} V(\mathbf{b}(s)) d s\right)\right]=C\left|\mathbf{x}-\mathbf{x}^{\prime}\right|^{-n+\rho}
\end{aligned}
$$

with a certain constant $C$ and

$$
\rho=(2-m)(1-\alpha+\beta)^{-1}
$$


For canonical quantum fields in $n$ dimensions we should have $\rho=2$. This happens if (in addition to eq.(24))

$$
\alpha-\beta=\frac{m}{2}
$$

In such a case the potential is

$$
V(\mathbf{y})=|\mathbf{y}|^{-m}
$$

The potential (32) scales in the same way as the $\delta$-function in $m$-dimensions. This is a singular potential. However, its regularization $V_{\epsilon}(\mathbf{y})=|\mathbf{y}|^{-m-\epsilon}$ for any $\epsilon>0$ gives a self-adjoint Hamiltonian with the well-defined path integral. As $\epsilon$ can be arbitrarily small the Newton potential on the brane would be indistinguishable from $r^{-1}$ if the brane is $n-1=3$ dimensional. We could again consider the limit $V(\mathbf{y}) \rightarrow \delta(\mathbf{y})$ in order to derive the model of Dvali et al [3]. In contradistinction to the case $m=1$ the models in $m>1$ dimensions are more complicated. For $m=2$ and $m=3$ the relation of the coupling constant $\mathbf{p}^{2}$ in eq.(26) to the parameters appearing in the heat kernel $K^{\delta}$ is not so explicit [15]. For $m>3$ the $\delta$-potential cannot be defined at all [16] [13].

We have discussed only scale invariant metrics. If the metric is not scale invariant but its asymptotic behaviour for $\mathbf{y} \rightarrow 0$ is of the form (22) then our results hold true when $-1 \leq \nu \leq 0$ and when applied to the short distance behaviour $\left|\mathbf{x}-\mathbf{x}^{\prime}\right| \rightarrow 0$ of $G\left(\mathbf{0}, \mathbf{x} ; \mathbf{0}, \mathbf{x}^{\prime}\right)$. If the asymptotic behaviour of the metric for $|\mathbf{y}| \rightarrow \infty$ is of the form (22) then our results apply if $\beta \geq \alpha$ to the behaviour of the Green's functions $G\left(\mathbf{0}, \mathbf{x} ; \mathbf{0}, \mathbf{x}^{\prime}\right)$ for large $\left|\mathbf{x}-\mathbf{x}^{\prime}\right|$. In such a case $\rho<2$ in eq. (30), hence $G\left(\mathbf{0}, \mathbf{x} ; \mathbf{0}, \mathbf{x}^{\prime}\right)$ and the gravitational potential decay to zero faster than in the Newton theory (in the $n$ dimensions). Depending on the asymptotic behaviour of the metric tensor $g(\mathbf{x}, \mathbf{y})$ we obtain models which lead to a modification of the Newton law either at small or at large distances ( some brane models modifying the classical gravity at small or large distances are discussed in [4][7]).

\section{References}

[1] G. Dvali, G. Gabadadze and M. Porrati, Phys.Lett.B485,208(2000)

[2] L. Randal and R. Sundrum, Phys.Rev.Lett.833370(1999);83,4690(1999)

[3] G. Dvali and G. Gabadadze, Phys.Rev.D63,065007(2001)

[4] R. Gregory, V.A. Rubakov and S.M. Sibiriakov, Phys.Rev.Lett. 84,5928(2000)

[5] G. Dvali, G. Gabadadze, X. Hou and E. Sefusatti, Phys.Rev.D67,044019(2003) 
[6] M. Mintchev and L.Pilo, Nucl. Phys.B592,219(2001); arXiv: hep-th/0007002

[7] S. B. Giddings, E. Katz and L. Randall, JHEP 0003(2000)023; arXiv: hepth/0002091

J. Garriga and T. Tanaka, Phys.Rev.Lett.84,2778(2000); arXiv:hep-th/9911055

I.I. Kogan and G.G. Ross, Phys.Lett.B485,255(2000)

[8] P. Szekeres and V. Iyer, Phys.Rev.D47,4362(1993)

M. Celerier and P. Szekeres, Phys.Rev.D65,123516(2002)

[9] G.W. Gibbon and D.L. Wiltshire, Nucl.Phys.B287,717(1986) arXiv:hepth/0109093

M.Duff and J. Lu, Nucl. Phys.B354,141(1991)

G. Horowitz and A. Strominger, Nucl.Phys. B360,197(1991)

A. Tseytlin, Nucl. Phys. B475,149(1996)

[10] G. Papadopoulos, arxiv:hep-th/0404172

[11] B. Simon, Functional Integration and Quantum Physics, Academic Press, 1979

[12] H. Ezawa, J.R. Klauder and L.A. Shepp, J.Math.Phys.16,783(1975)

[13] S. Albeverio, F. Gesztesy, R. Hoegh-Krohn and H. Holden, Solvable Models in Quantum Mechanics, Springer,Berlin,1988

[14] B. Gaveau and L. Schulman, J. Phys.A19,1833(1986)

[15] S. Albeverio, Z. Brzezniak and L. Dabrowski, J.Phys. A27,4933(1994)

[16] F.A. Berezin and L.D. Faddeev, Soviet Math.Dokl.2,372(1961) 